\title{
(2) Association of mammographic density and OPEN ACCESS benign breast calcifications individually or combined with hypertension, diabetes, and hypercholesterolemia in women $\geq 40$ years of age: a retrospective study
}

\author{
Maha Al-Mohaissen (ㄷ, ${ }^{1}$ Arwa Alkhedeiri, ${ }^{2}$ Ohoud Al-Madani, ${ }^{3}$ Terry Lee, ${ }^{4}$ \\ Anas Hamdoun, ${ }^{2}$ Mohammad Al-Harbi ${ }^{2}$
}

'Department of Clinical Sciences (Cardiology), College of Medicine, Princess Nourah bint Abdulrahman University, Riyadh, Saudi Arabia

2Department of Radiology, King Abdullah Bin Abdulaziz University Hospital, Riyadh, Saudi Arabia

${ }^{3}$ Department of Research Informatics, Saudi Food and Drug Authority, Riyadh, Saudi Arabia

${ }^{4}$ Centre for Health Evaluation and Outcome Sciences, Vancouver, British Columbia, Canada

\section{Correspondence to} Dr Maha Al-Mohaissen, Department of Clinical Sciences (Cardiology), College of Medicine, Princess Nourah bint Abdulrahman University, P.0. Box 84428 , Riyadh 11671, Saudi Arabia; maalmohaissen@pnu.edu.sa

Accepted 26 January 2022

Check for updates

(c) American Federation for Medical Research 2022. Re-use permitted under CC BY-NC. No commercial re-use. Published by BMJ.

To cite: Al-Mohaissen $\mathrm{M}$, Alkhedeiri A, Al-Madani O et al. J Investig Med Epub ahead of print: [please include Day Month Year]. doi:10.1136/jim-2021002296

\section{ABSTRACT}

Recent evidence has linked certain mammographic characteristics, including breast calcifications (Bcs) and mammographic density (MD), with atherosclerotic cardiovascular disease risk factors in women, but data are limited and inconsistent. We aimed to evaluate the association of MD and/or Bcs with hypertension, diabetes, and hypercholesterolemia in women $\geq 40$ years of age. Through hospital electronic records, we retrospectively identified mammograms of nonpregnant women aged $\geq 40$ years and without breast cancer and retrieved reports and relevant data. $M D$ and Bcs were recorded; risk factor status was diagnosed based on treatment profile and clinical and laboratory data. In total, 1406 women were included. MD was inversely related to hypertension, diabetes, hypercholesterolemia, triglyceride levels, age, and body mass index (BMI) ( $p$ value for trend $<0.001)$. Bcs were positively associated with hypertension, diabetes, hypercholesterolemia, age, $B M I$, and elevated creatinine $(p<0.05)$. Controlling for age and BMI, MD category A (MD-A) was independently associated with hypercholesterolemia; Bcs were independently associated with diabetes. Combining MD-A with Bcs did not increase the odds significantly. Analysis for additive interactions revealed a significant interaction between MD-A and BMI, increasing the odds of hypertension, and a trend for increased odds of diabetes by adding MD$A$ and/or Bcs to BMI. Decreased MD and presence of Bcs are associated with hypertension, diabetes, and hypercholesterolemia in women $\geq 40$ years of age. MD-A may represent a new obesity index independently associated with hypercholesterolemia and additive to hypertension risk. Bcs are independently associated with diabetes. Combining MD and Bcs did not improve the odds significantly, which may reflect mechanistic differences.

\section{INTRODUCTION}

Recent evidence has linked some breast tissue components as viewed on mammographic

\section{Significance of this study}

What is already known about this subject?

- There is an increasing interest in extending the benefits of screening mammography to include cardiovascular disease (CVD) screening and risk stratification.

- Studies favor an association between breast arterial calcifications and CVD and CVD risk factors.

- Few studies examined the association of other breast tissue components including mammographic density (MD) with CVD or CVD risk factors.

What are the new findings?

- We evaluated and compared the associations of MD and benign breast calcifications (BCs) with hypertension, hypercholesterolemia, and diabetes in women $\geq 40$ years of age.

- Both MD and Bcs were significantly associated with these three risk factors.

- Controlling for age and body mass index (BMI), Bcs were independently associated with 1.4-fold increased odds of diabetes.

- MD category A was independently associated with 1.6-fold increased odds of hypercholesterolemia, and when added to BMI more than doubled the odds of hypertension.

- MD category A is related to increased BMI and parallels its association with the risk factors, and may represent a new obesity index in women $\geq 40$ years of age.

How might these results change the focus of research or clinical practice?

- The additional information gained from the mammogram regarding CVD risk factors may identify opportunities for targeted atherosclerotic CVD risk factor screening, early treatment, and prevention, and add to CVD risk stratification among women $\geq 40$ years of age. 
examinations with atherosclerotic cardiovascular disease (CVD) risk factors in women. ${ }^{1}$ Among those, benign breast calcifications (Bcs) are better studied, although data remain limited. $^{2}$ However, mammographic examinations report on other breast tissue characteristics that remain underexplored in that regard. In particular, mammographic density (MD), which classifies breast composition according to the amount of fatty and fibroglandular tissue, ${ }^{3}$ has been extensively studied in relation to breast cancer risk, ${ }^{4}$ but its association with other vital issues related to women's health, particularly CVD health, remains to be explored.

Since a majority of women who are 40 years of age or older undergo mammography annually for screening for breast cancer, further evaluation of mammogram images may provide additional information regarding important CVD risk factors. The additional details may provide insights regarding screening and prevention of CVD risk factors, and CVD risk stratification at no extra cost or radiation, and increase the test yield. ${ }^{1}$ In view of the shortage and inconsistency of available data, this study aimed to evaluate the association of MD and Bcs individually and combined with the risk of hypertension, diabetes, and hypercholesterolemia in a large population of women $\geq 40$ years of age. The association of MD and Bcs with other lipid parameters, including triglycerides and high-density lipoprotein cholesterol (HDL-C), was also evaluated.

\section{METHODS}

\section{Study design and patient population}

Women who underwent a mammogram at King Abdullah bin Abdulaziz University Hospital, Riyadh, Saudi Arabia, from March 2017 to March 2020 were retrospectively identified through the hospital database. Participants were considered eligible if they were $\geq 40$ years of age and had determinable blood pressure (BP), cholesterol, or glycemic status from the electronic hospital records. The exclusion criteria were breast cancer, uninterpretable images, and pregnancy. If a participant had multiple mammograms over the years, the most recent or the one with the most complete data of interest was included.

\section{Data collection and diagnostic criteria}

For the diagnosis of hypertension, hypercholesterolemia, and diabetes, an electronic search was performed to retrieve participants' medications and relevant investigation results (fasting lipid profile, fasting or random blood sugar (FBS or RBS), and hemoglobin A1c (HbA1c)). For BP data, a manual search for non-emergency, outpatient BP readings was performed. Women's age, height and weight for body mass index (BMI) calculation, and creatinine levels were also recorded. Only results within 1 year of the mammogram date and the closest (if multiple) were included.

Participants were identified as hypertensive if they were receiving antihypertensive medications or their BP reading met the thresholds for hypertension, ${ }^{5}$ and normotensive if the untreated BP was $<130 / 80 \mathrm{~mm} \mathrm{Hg}$. Hypercholesterolemia was diagnosed if the participant was receiving statin therapy or had a fasting low-density lipoprotein cholesterol (LDL-C) $\geq 4.9 \mathrm{mmol} / \mathrm{L}^{6}$; its absence was indicated by untreated LDL-Clevel $<4.9 \mathrm{mmol} / \mathrm{L}$. Diabetes was diagnosed if the participant was receiving antidiabetic medications or had at least one FBS, RBS, or HbA1c level meeting the diagnostic criteria for diabetes (FBS $\geq 7.0 \mathrm{mmol} / \mathrm{L}$, RBS $\geq 11.1 \mathrm{mmol} / \mathrm{L}$, or $\mathrm{HbA} 1 \mathrm{c} \geq 6.5 \%),{ }^{7}$ while euglycemia was indicated by untreated euglycemic indices. The records of the participants who were receiving only a small dose of a beta-blocker, ACE inhibitor, angiotensin receptor blocker, metformin, or a glucagon-like peptide-1 receptor agonist were searched for indications. If the indications were other than hypertension or diabetes, they were classified based on their BP and blood sugar index results.

MD category was recorded as defined by the American College of Radiology's Breast Imaging Reporting and Data System (Fifth Edition): category A (MD-A): almost entirely fatty breast tissue; $\mathrm{B}(\mathrm{MD}-\mathrm{B})$ : scattered fibroglandular densities; C (MD-C): heterogeneously dense breast tissue; and D (MD-D): extremely dense. ${ }^{8}$ The presence or absence of Bcs was also noted and these were classified according to size as microcalcifications $(\leq 2 \mathrm{~mm})$ and macrocalcifications $(>2 \mathrm{~mm}){ }^{9}$

\section{Statistical analysis}

All data obtained were recorded on Excel spreadsheets and in SAS 9.4. Continuous variables are reported as mean and SD or median and IQR, as appropriate, while categorical variables are expressed as frequencies with percentages. Statistical significance was defined as $p<0.05$. Logistic regression adjusted for age and BMI was used to examine the association between major CVD risk factors (BP status, hypercholesterolemia, and diabetes status) and participant characteristics (MD-A vs non$\mathrm{MD}-\mathrm{A}$, and presence of Bcs) in the entire cohort. We then further conducted an analysis adjusted for age and stratified by obesity status $\left(\mathrm{BMI}>30 \mathrm{~kg} / \mathrm{m}^{2}\right)$. A saturated logistic regression model that included all two-way and three-way interactions between MD, Bcs, and obesity was used to estimate the association in each stratum defined by the combination of these three variables. The results are presented as ORs and their 95\% CIs.

\section{RESULTS}

\section{Selection of the study participants}

In total, 1406 women met the study criteria (figure 1). Among these, 32 were receiving small doses of betablockers, ACE inhibitors, or angiotensin receptor blockers for indications other than hypertension (palpitations, coronary artery disease (CAD), or renal disease); they were

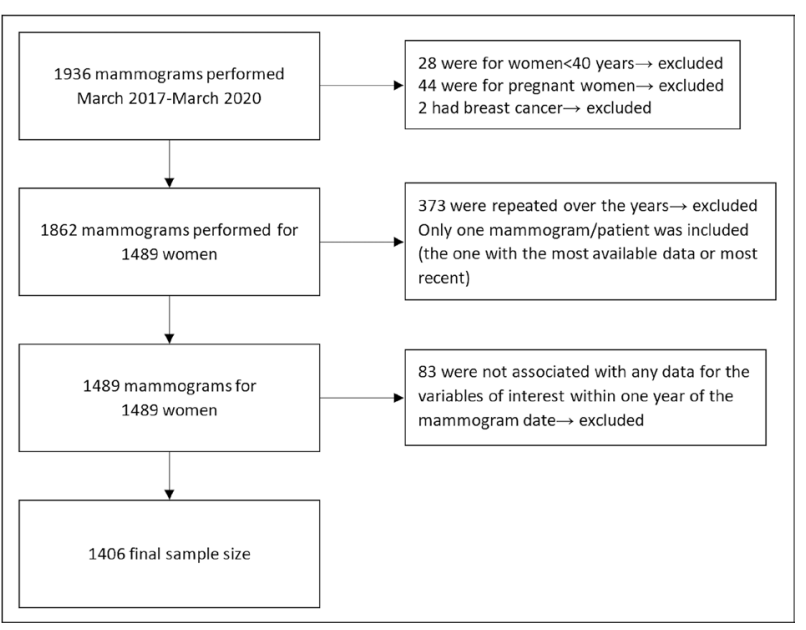

Figure 1 Selection of the study participants. 
analyzed according to their BP values. Another 83 were receiving metformin or liraglutide for polycystic ovary syndrome, obesity, or pre-diabetes and were analyzed according to their glycemic parameters. These two groups were evenly distributed among the MD and Bcs categories.

\section{Baseline characteristics of the participants}

The baseline characteristics of the study population are displayed in table 1 . The mean age was 53.8 years. Overall, 8.9\% had MD-A, and 38.8\%, 47.7\%, and 4.6\% had MD-B, MD-C, and MD-D, respectively. Bcs were detected in $20 \%$. Approximately $55.3 \%$ and $38.6 \%$ had stage 1 and stage 2 hypertension, respectively. Hypercholesterolemia was observed in $48.1 \%$ of patients and $32.3 \%$ had diabetes. The results of HDL-C and triglycerides are displayed.

\section{Relationship between MD and Bcs and the studied variables}

The relationship between MD and Bcs and the studied variables is shown in table 1 . Significant negative trends were detected between MD category and participants' age, BMI, hypertension, hypercholesterolemia, diabetes, and mean triglyceride level $(p<0.001)$. Those in the lowest MD category (MD-A) were older and had greater BMI and risk factor prevalence (hypertension: 69.9\%, hypercholesterolemia: $65.4 \%$, and diabetes: $43.6 \%$ ). There was no significant difference in HDL-C (mean or $<1.3 \mathrm{mmol} / \mathrm{L}$ ), hypertriglyceridemia (triglycerides $\geq 2 \mathrm{mmol} / \mathrm{L}$ ), or creatinine level between the four MD categories.

In the case of Bcs, the presence of any Bcs was associated with increasing age $(p<0.001)$, BMI $(p=0.043)$, hypertension $(p=0.008)$, hypercholesterolemia $(p=0.016)$, diabetes $(p<0.001)$, elevated creatinine $(p<0.001)$, and mean triglyceride level $(p=0.021)$, but not triglycerides $\geq 2 \mathrm{mmol} / \mathrm{L}$ or HDL-C measures. When Bcs were stratified according to size, significant positive trends for increased prevalence of hypertension $(\mathrm{p}=0.012)$, hypercholesterolemia $(p=0.010)$, and diabetes $(p<0.001)$ with increasing Bcs size were detected. Bcs were more prevalent in women with MD-D. A significant positive trend between increasing $\mathrm{MD}$ and Bcs was detected $(\mathrm{p}=0.006)$. The distribution of MD and/or Bcs per obesity status in the study population and the distribution of the studied risk factors among participant characteristics and among all participants with the same risk factor are shown in figure 2.

The evaluation of the association of $\mathrm{MD}$ and/or Bcs with the studied risk factors after adjustment for BMI and age is shown in figure 3. Neither MD-A nor Bcs individually or combined was independently associated with hypertension risk. Conversely, the odds of hypercholesterolemia were significantly increased in women with MD-A (OR 1.60, $95 \%$ CI 1.00 to $2.54, p=0.048$ ), and the presence of any Bcs independently increased the odds of diabetes (OR 1.4, $95 \%$ CI 1.01 to $1.94, \mathrm{p}=0.043$ ).

\section{Evaluation for additive interactions between MD-A and/ or Bcs with obesity}

Further assessment for additive interactions between MD-A and/or Bcs with obesity on the odds of the three risk factors, with adjustment for age, is shown in figure 4. Compared with non-obese women with non-MD-A densities and no
Bcs (the reference), obese women with non-MD-A densities and no Bcs (obese only) had significantly increased odds of hypertension (OR $2.09,95 \%$ CI 1.6 to $2.74, p<0.001$ ). The addition of MD-A to obesity increased the odds of hypertension significantly more than obesity alone (OR 5.37 compared with $2.09, \mathrm{p}=0.005)$. The addition of calcification to obesity did not increase the odds of hypertension compared with obesity only (OR 2.27 compared with 2.09, $\mathrm{p}=0.686$ ). Conversely, the addition of both Bcs and MD-A to obesity decreased the odds of hypertension compared with MD-A and obesity, although this was statistically insignificant (OR 5.09 compared with 5.37, $\mathrm{p}=0.949$ ).

With regard to hypercholesterolemia, obese women with non-MD-A densities and no Bcs (obese only) had increased odds for dyslipidemia compared with the reference (OR $1.36,95 \% \mathrm{CI} 1.01$ to $1.84, \mathrm{p}=0.041$ ). The addition of MD-A to obesity increased the odds of hypercholesterolemia compared with the reference (OR 2.22, 95\% CI 1.20 to $4.11, \mathrm{p}=0.011$ ) but not compared with obesity only (OR 2.22 compared with 1.36, $\mathrm{p}=0.115$ ). Neither the addition of Bcs nor Bcs and MD-A was associated with a significant increase in the odds of hypercholesterolemia compared with the reference.

In the case of diabetes, the odds of all four comparisons were significantly higher than the reference, with a tendency for a graded increase. However, compared with the odds of obesity only, the addition of MD-A to obesity did not significantly increase the odds of diabetes (OR 2.80 compared with $2.59, \mathrm{p}=0.797)$. Conversely, the addition of Bcs or Bcs and MD-A to obesity resulted in trends for increased odds of diabetes compared with obesity only (OR 3.56 compared with $2.59, \mathrm{p}=0.129$ and OR 5.02 compared with $2.59, \mathrm{p}=0.362$, respectively), but they did not reach statistical significance.

\section{DISCUSSION}

The results of this study show that in women $\geq 40$ years of age, both MD and Bcs can be linked to the risk of hypertension, diabetes, and hypercholesterolemia, but with variable associations and strengths. A significant inverse trend between MD category and the three risk factors was observed, with women falling in the lowest category or MD-A having the highest prevalence. After adjustments for BMI and age, MD-A was independently associated with a 1.6-fold increase in the odds of hypercholesterolemia. No independent association was observed with hypertension or diabetes. Similarly, the presence of any Bcs was associated with an increased prevalence of the three risk factors, with a graded increase with increased Bcs size. Controlling for BMI and age, Bcs were independently associated with a 1.4fold increase in the odds of diabetes.

Analysis for additive interactions detected more than doubling of the odds of hypertension by the addition of MD-A to obesity compared with obesity only and a statistically insignificant increase in the odds of hypercholesterolemia. The addition of Bcs to MD-A and obesity did not further increase the odds of hypertension or hypercholesterolemia significantly. Conversely, the addition of MD-A, Bcs, or both to obesity resulted in a tendency for a graded increase in the risk of diabetes; however, this did not reach statistical significance, which may be due to the 


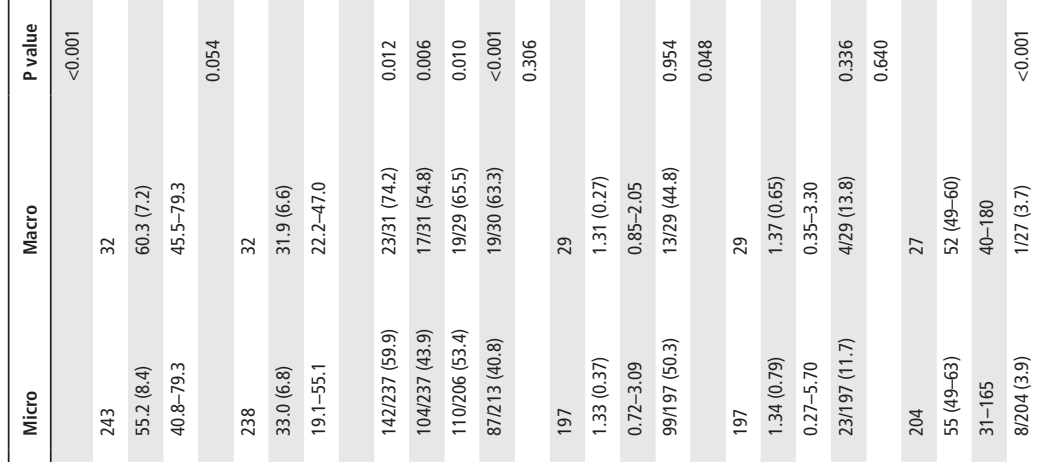

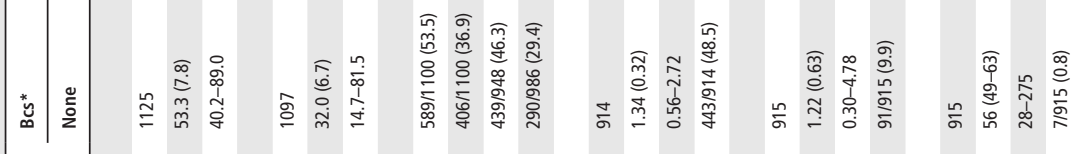

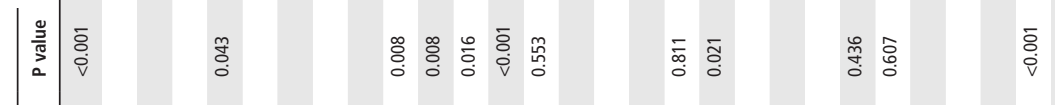

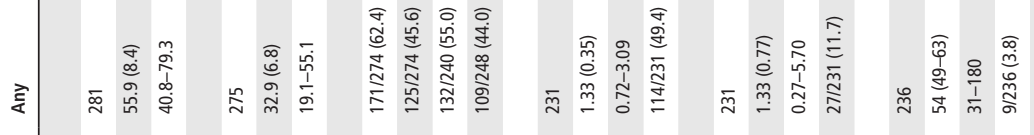

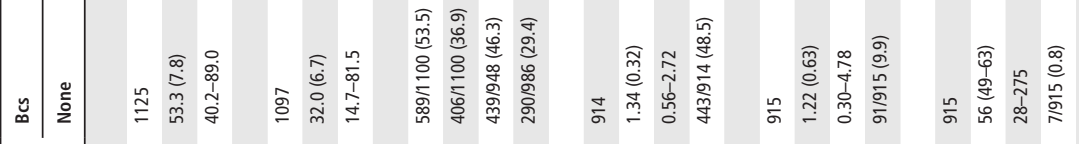

|

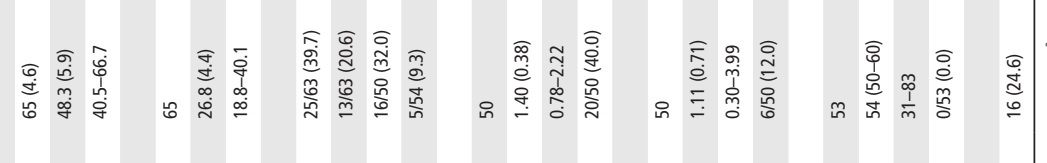

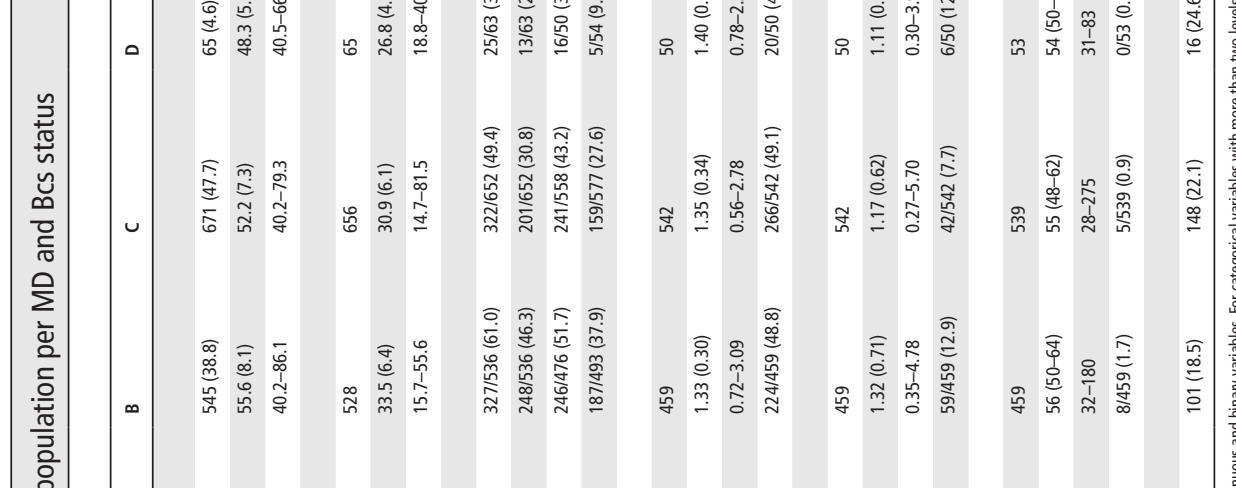

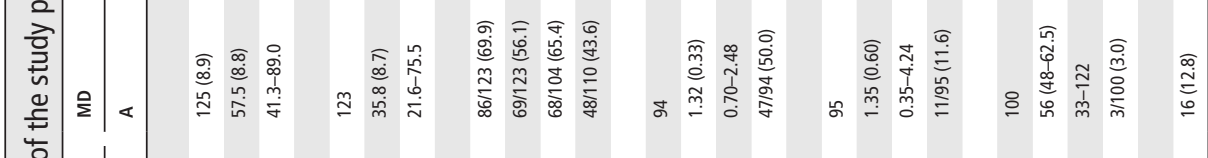

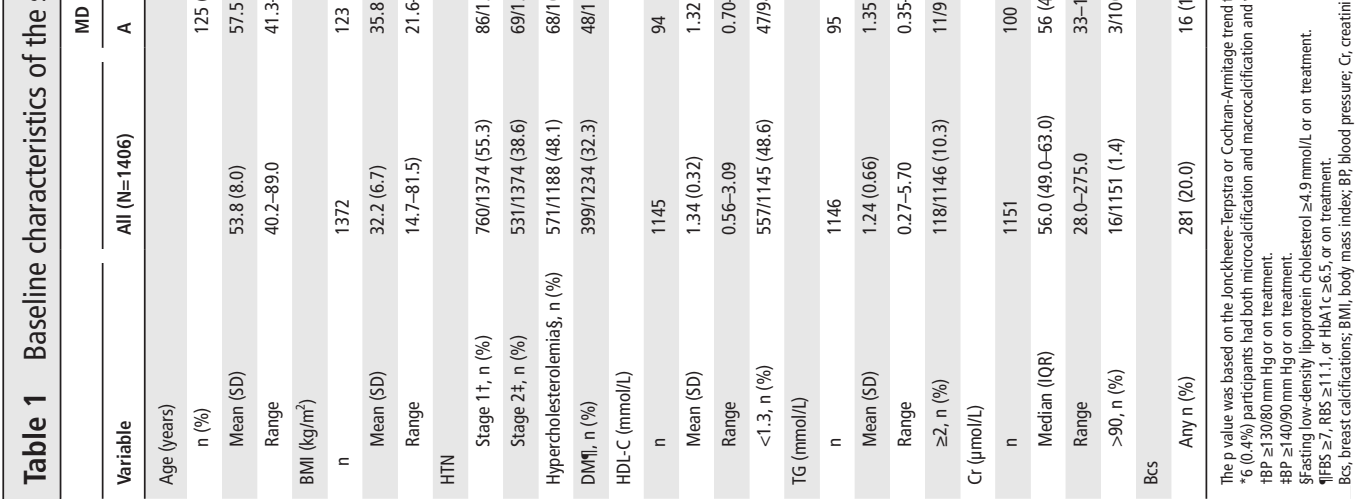




\begin{tabular}{|c|c|c|c|c|c|c|c|c|c|}
\hline \multicolumn{5}{|c|}{ Nonobese $n=579(42.2 \%)$} & \multicolumn{5}{|c|}{ Obese $n=793$ (57.8\%) } \\
\hline $\begin{array}{c}\text { Patient } \\
\text { characteristics }\end{array}$ & \begin{tabular}{|c|}
$\mathrm{N}(\%)$ of \\
total study \\
population
\end{tabular} & Risk factor & $\begin{array}{c}\mathbf{N}(\%) \text { among } \\
\text { patient } \\
\text { characteristics }\end{array}$ & $\begin{array}{l}\mathrm{N}(\%) \text { among } \\
\text { risk factor }\end{array}$ & $\begin{array}{c}\text { Patient } \\
\text { characteristics }\end{array}$ & \begin{tabular}{|c|}
$N(\%)$ of \\
total study \\
population
\end{tabular} & \begin{tabular}{|l|} 
Risk factor \\
\end{tabular} & $\begin{array}{c}\mathbf{N}(\%) \text { among } \\
\text { patient } \\
\text { characteristics }\end{array}$ & $\begin{array}{l}\mathbf{N}(\%) \text { among } \\
\text { risk factor }\end{array}$ \\
\hline $\begin{array}{l}\text { Nonobese } \\
\text { Non MD-A } \\
\text { No Bcs }\end{array}$ & $446(32.5)$ & $\begin{array}{l}\text { Hypertension } \\
\text { Hypercholesterolemia } \\
\text { Diabetes }\end{array}$ & $\begin{array}{l}175 / 437(40.0) \\
140 / 369(37.9) \\
68 / 390(17.4)\end{array}$ & \begin{tabular}{|c|}
$175 / 742(23.6)$ \\
$140 / 553(25.3)$ \\
$68 / 389(17.5)$
\end{tabular} & \begin{tabular}{|l} 
Obese \\
Non MD \\
No Bcs
\end{tabular} & $544(39.7)$ & $\begin{array}{l}\text { Hypertension } \\
\text { Hypercholesterolemia } \\
\text { Diabetes }\end{array}$ & $\begin{array}{l}325 / 534(60.9) \\
227 / 464(48.9) \\
174 / 472 \text { (36.9) }\end{array}$ & $\begin{array}{l}325 / 742(43.8) \\
227 / 553(41.0) \\
174 / 389(44.7)\end{array}$ \\
\hline $\begin{array}{l}\text { Nonobese } \\
+ \text { MD-A }\end{array}$ & $31(2.3)$ & $\begin{array}{l}\text { Hypertension } \\
\text { Hypercholesterolemia } \\
\text { Diabetes }\end{array}$ & $\begin{array}{l}11 / 31(35.5) \\
13 / 25(52.0) \\
7 / 28(25.0)\end{array}$ & $\begin{array}{l}11 / 742(1.5) \\
13 / 553(2.4) \\
7 / 389(1.8)\end{array}$ & $\begin{array}{l}\text { Obese } \\
+ \text { MD-A }\end{array}$ & $76(5.5)$ & \begin{tabular}{|l} 
Hypertension \\
Hypercholesterolemia \\
Diabetes
\end{tabular} & $\begin{array}{l}63 / 75(84.0) \\
44 / 64(68.8) \\
33 / 68(48.5)\end{array}$ & $\begin{array}{l}63 / 742(8.5) \\
44 / 553(8.0) \\
33 / 389(8.5)\end{array}$ \\
\hline $\begin{array}{l}\text { Nonobese } \\
+ \text { Bcs }\end{array}$ & $98(7.1)$ & $\begin{array}{l}\text { Hypertension } \\
\text { Hypercholesterolemia } \\
\text { Diabetes }\end{array}$ & $\begin{array}{l}48 / 95(50.5) \\
44 / 82(53.7) \\
25 / 86(29.1)\end{array}$ & $\begin{array}{l}48 / 742(6.5) \\
44 / 553(8.0) \\
25 / 389(6.4)\end{array}$ & $\begin{array}{l}\text { Obese } \\
+ \text { + Bcs }\end{array}$ & $161(11.7)$ & $\begin{array}{l}\text { Hypertension } \\
\text { Hypercholesterolemia } \\
\text { Diabetes }\end{array}$ & $\begin{array}{l}108 / 158(68.4) \\
76 / 140(54.3) \\
75 / 146(51.4)\end{array}$ & $\begin{array}{l}108 / 742(14.6) \\
76 / 553(13.7) \\
75 / 389(19.3)\end{array}$ \\
\hline $\begin{array}{l}\text { Nonobese } \\
+ \text { MD-A } \\
+ \text { Bcs }\end{array}$ & $4(0.3)$ & $\begin{array}{l}\text { Hypertension } \\
\text { Hypercholesterolemia } \\
\text { Diabetes }\end{array}$ & $\begin{array}{l}2 / 4(50.0) \\
1 / 3(33.3) \\
1 / 2(50.0)\end{array}$ & $\begin{array}{l}2 / 742(0.3) \\
1 / 553(0.2) \\
1 / 389(0.3)\end{array}$ & $\begin{array}{l}\text { Obese } \\
+ \text { + MD-A } \\
+ \text { Bcs }\end{array}$ & $12(0.9)$ & $\begin{array}{l}\text { Hypertension } \\
\text { Hypercholesterolemia } \\
\text { Diabetes }\end{array}$ & $\begin{array}{l}10 / 12(83.3) \\
8 / 10(80.0) \\
6 / 10(60.0)\end{array}$ & $\begin{array}{l}10 / 742(1.3) \\
8 / 553(1.4) \\
6 / 389(1.5)\end{array}$ \\
\hline
\end{tabular}

Figure 2 Distribution of MD-A and/or Bcs per obesity status in the study population and distribution of the studied risk factors among participant characteristics and among all participants with the same risk factor. The presence of MD-A and/or Bcs, although more commonly observed among obese women, represented $<10 \%$ and $<20 \%$ of the non-obese and obese populations, respectively. Bcs, benign breast calcifications; MD-A, mammographic density A.

small number of participants with this cluster. These findings add to the value of the mammogram in women's health and support personalized patient-provider discussions of the mammogram findings ${ }^{1}$ beyond breast cancer risk. If Bcs or reduced $\mathrm{MD}$, particularly $\mathrm{MD}-\mathrm{A}$, is detected, screening for CVD risk factors, if not already attempted, should take place and closer follow-up and intensification of healthy lifestyle changes should be advised.

\section{Association of MD-A with the studied CVD risk factors}

In this study, we evaluated the associations of MD from a metabolic perspective, while most previous literature

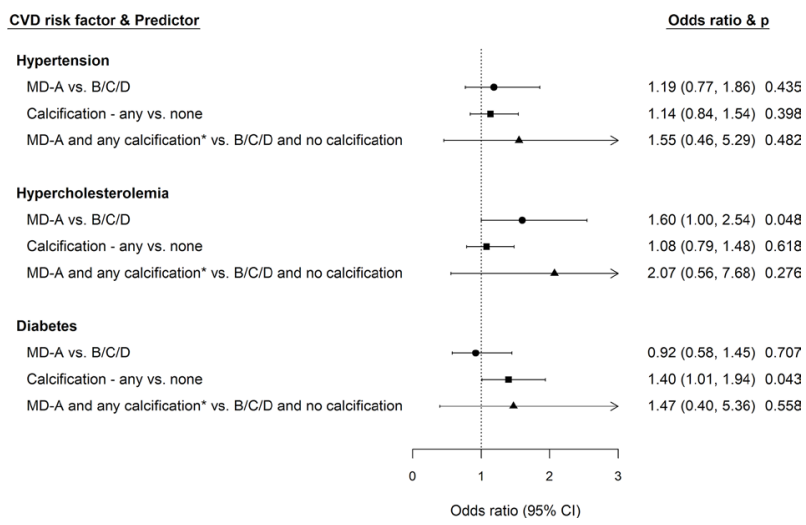

Figure 3 OR for the studied risk factors per mammographic density and breast calcifications with adjustment for body mass index and age. * $\mathrm{n}$ is only 16 for hypertension, 13 for hypercholesterolemia, and 12 for diabetes mellitus. CVD, cardiovascular disease; MD, mammographic density; MD-A, almost entirely fatty breast tissue; MD-B, scattered fibroglandular densities; MD-C, heterogeneously dense breast tissue; MDD,extremely dense. reported from a breast cancer risk point of view. ${ }^{10} 11$ We observed that MD-A was closely related to increased BMI, and both were related in a similar manner to the studied risk factors. This parallel relationship of $\mathrm{MD}-\mathrm{A}$ and $\mathrm{BMI}$ with the risk factors suggests that MD-A or increased breast adiposity may represent a new non-anthropometric obesity or body fat distribution index ${ }^{12-14}$ that can be linked to atherosclerotic risk factors among women. Although obesity contributes directly to incident CVD risk factors, it is the regional body fat distribution, which is often sex-related, that is largely responsible for the observed differences in risk profiles. ${ }^{15}{ }^{16}$ Breast adipose tissue is an endocrine organ that secretes adipokines, cytokines, chemokines, and growth factors. ${ }^{17}$ Women with increased body fat exhibit greater breast inflammation with higher levels of circulating metaboinflammatory factors, ${ }^{11}$ and greater expression of

CVD risk factor \& Predictor
Hypertension
Obese only
Obese + MD-A
Obese + Bcs
Obese + MD-A + Bcs
Hypercholesterolemia
Obese only
Obese + MD-A
Obese + Bcs
Obese + MD-A + Bcs
Diabetes
Obese only
Obese + MD-A
Obese + Bcs
Obese + MD-A + Bcs

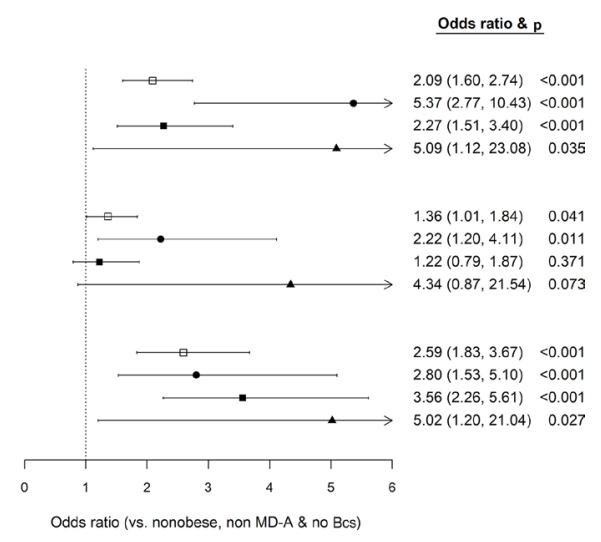

Figure 4 OR of the studied risk factors per MD-A and/or Bcs added to obesity, with adjustment for age. Bcs, benign breast calcifications; CVD, cardiovascular disease; MD-A, mammographic density $A$. 
adipogenic and proinflammatory genes is detected in the breast adipose tissue of obese women compared with nonobese women. ${ }^{18}$

Limited available evidence has focused on the association of MD with the metabolic syndrome cluster ${ }^{1019-23}$ rather than individual CVD risk factors. Inconsistencies in the results are noted, likely due to the small number of participants, ${ }^{102124}$ inclusion of variable age groups (premenopausal, ${ }^{22}$ postmenopausal, ${ }^{21}$ or both ${ }^{19}$ ), and multiple ethnicities. ${ }^{10} 24$ However, available data, in similar-aged populations, have hinted of an inverse association between MD and metabolic syndrome, particularly atherogenic lipid profiles, which supports our observations. In a cross-sectional study from Thailand involving 713 women $\geq 40$ years of age, an inverse association between metabolic syndrome and per cent MD was noted, and after controlling for BMI the inverse association remained with triglycerides. ${ }^{23}$ Tehranifar $e t ~ a l,{ }^{24}$ in a study involving 191 women 40 years of age or older, found that only a history of hypercholesterolemia was inversely associated with per cent density and dense area. Having multiple metabolic conditions was also associated with lower density. In another study by the same group, however, no association between BMI-adjusted per cent density and metabolic syndrome or its components was detected in a similar-aged population, apart from HDL-C, which was associated with increased density. ${ }^{10}$ Conversely, in a study involving 364 premenopausal Chilean women, no association between metabolic syndrome and absolute dense volume was found; however, triglycerides were related to higher absolute dense volume. ${ }^{20}$ In the largest study to date, involving 73,974 Korean women, metabolic syndrome and its components were associated with dense breasts (MD-D) in premenopausal women, while in both premenopausal and postmenopausal women high blood glucose and insulin resistance were positively associated with dense breasts. ${ }^{22}$ Our findings on MD have validity; the distribution of MD categories $^{25-27}$ in this study and the inverse relation between $\mathrm{MD}$ and age and $\mathrm{BMI}^{2527-29}$ are consistent with the available literature. Further, the registered prevalence of the studied risk factors is in keeping with reports from Saudi Arabia and the world. ${ }^{5031}$ The relation between MD-A and the three risk factors is in harmony with what is anticipated from its established association with BMI.

The observed association of MD-A with hypercholesterolemia confirms earlier reports hinting at an association. ${ }^{2324}$ The stronger association in the case of hypercholesterolemia compared with the other risk factors may be explained by the fact that hypercholesterolemia is a classic characteristic of obesity ${ }^{32}$ and is the most common obesity-related comorbidity, followed by hypertension and diabetes. ${ }^{33}$ It is well established that changes in body fat depots significantly impact plasma lipoprotein composition and concentrations. ${ }^{32}$ The independent association of MD-A with hypercholesterolemia suggests that MD-A may increase cholesterol levels through metabolic pathways that are not related to obesity. Evidence from bariatric surgery studies has shown that the metabolic effects of bariatric surgery on the breast parenchyma and MD are independent of absolute BMI reduction, suggesting the existence of such pathways. ${ }^{34}$ While no similar mammographic data exist for comparison, the independent association of hypercholesterolemia with other obesity indices supports our findings. ${ }^{35}$
In the case of hypertension, the significant additive interaction between MD-A and BMI is in accordance with other investigators' observations that the combination of obesity indices and BMI yields the highest risk of incident hypertension compared with each measure alone. ${ }^{3637}$ Unlike waist circumference, ${ }^{14}$ however, MD provided an additive but not independent association with hypertension. Conversely, on the other end of the spectrum, increased gluteofemoral adiposity decreases metabolic disease prevalence. ${ }^{14}{ }^{38}$ It may be postulated that MD-A is an intermediate between the two other phenotypes. Breast adipose-derived stem cells exhibit greater immunomodulatory and antioxidative capabilities than their abdominal counterparts. ${ }^{39}$ There are no comparisons with gluteofemoral adipose cells at present. The association of MD-A with hypertension and hypercholesterolemia adds to the present knowledge of the differences in obesity-associated hypertension and hypercholesterolemia in men and women, which is of immense clinical importance. ${ }^{40} 41$

The association of MD-A with diabetes was less pronounced. Obesity and obesity indices have strong associations with diabetes ${ }^{42}$; in this study, however, we did not detect an independent association between diabetes and MD-A and only a trend for increased odds of diabetes was observed by the addition of MD-A to obesity. This may be due to the fact that diabetes, unlike the other studied risk factors, has a dual effect on MD, causing both reduced and increased $\mathrm{MD},{ }^{43}$ thereby compromising the significance of the association. Increases in MD have been linked to diabetes per se, ${ }^{2244}$ its complications, ${ }^{45}$ and insulin therapy. ${ }^{43}$

\section{Association of Bcs with the studied CVD risk factors}

Although we evaluated benign Bcs in general, which may have various etiologies, ${ }^{9}$ women with diabetes tend to develop breast arterial calcifications (BACs), which involve the medial layer of the arterioles. ${ }^{146}$ The observed predictors of Bcs in this study and the independent association with diabetes are in accordance with current literature on BACs. ${ }^{1}$ While all major CVD risk factors have been linked to the development/presence of arterial calcifications, diabetes appears to be the most important predisposing factor ${ }^{4}{ }^{4}$ which is in accordance with the study findings.

The present evidence of the association of BACs with CVD risk factors is indirectly derived from studies evaluating its association with CVD and reproductive factors in addition to CVD risk factors. ${ }^{48} 49$ recent meta-analysis involving studies that assessed the association of BACs with CAD found that BACs were positively associated with diabetes, hypertension, and hypercholesterolemia. When only good-quality studies were included, the association with hypercholesterolemia became insignificant. ${ }^{48}$ In another meta-analysis, when good-quality and multivariable adjusted studies were included, ORs of 1.56 (95\% CI 1.32 to 1.83 ) and 1.73 (95\% CI 1.39 to 2.15 ) were detected for diabetes, respectively. No independent association with hypertension or hypercholesterolemia was observed. ${ }^{49}$ Our results are in accordance with these meta-analyses and support them. This study, however, directly assessed the association of Bcs with the risk factors and was not subject to the methodological heterogeneity and selection bias that may occur from the inclusion of variable studies 
in meta-analyses. ${ }^{48} 49$ Additionally, to the best of our knowledge, Bcs have never been evaluated in comparison with or in addition to MD.

\section{Association of MD-A and Bcs combined with risk factors}

We observed no further increase in the odds of the risk factors by the addition of Bcs to MD-A and obesity, except for a tendency for increased odds of diabetes. While this may be due to the small number of participants with these characteristics, asynergy in the mechanisms behind the association of MD-A and Bcs with the risk factors is another potential explanation. The increased prevalence of Bcs in women with increased MD supports this theory.

The association of adiposity with the three studied risk factors is well established. Obesity contributes directly to the occurence of hypertension, diabetes, and dyslipidemia, ${ }^{16}$ and it may be speculated that a similar association occurs in the case of MD-A. Conversely, the association of arterial calcifications with CVD risk factors varies. Although hypertension, hypercholesterolemia, and diabetes have been associated with arterial calcifications, this relation is not consistent across all vascular beds and for men and women. ${ }^{50}$ In the case of BACs, an association with diabetes is reported but not with obesity, hypertension, or dyslipidemia. ${ }^{49}$ At the molecular level, the pathogenesis of arterial calcifications involves multiple biological pathways including those related to inflammation, apoptosis, disruptions of calcium phosphate homeostasis, extracellular matrix degeneration, and genetic aberrations, and the initial drivers remain unclear. ${ }^{46}$ Similarly, obesity has a complex pathogenesis and exhibits heterogeneity in the pathways and mechanisms by which it leads to adverse health outcomes. ${ }^{16}$ While some overlap in the pathways of adiposity and arterial calcifications may exist, their effects/ associations with the studied risk factors remain distinct. It may be that the association of MD-A with hypertension and hypercholesterolemia is a causal one emanating from its relation to obesity, while Bcs are a consequence of the inflammatory state commonly associated with insulin resistance and diabetes. ${ }^{47}$ The observed tendency for increased odds of diabetes by the addition of both Bcs and MD-A to obesity is likely due to its strong association with both obesity $^{42}$ and Bcs. ${ }^{47}$

\section{CONCLUSION}

We found that both MD and Bcs are significantly associated with atherosclerotic CVD risk factors. In particular, MD-A was independently associated with hypercholesterolemia and additive to BMI increasing hypertension risk and may represent a new obesity index in women. Bcs were independently associated with diabetes. These findings have clinical value. Since a majority of women above 40 years of age undergo mammography annually for breast cancer screening, additional information gained from the mammogram may identify opportunities for targeted atherosclerotic CVD risk factor screening, early treatment, and prevention, and add to CVD risk stratification among them.

Contributors MA-M: conceptualization, writing the initial draft, ethics and funding acquisition, data interpretation, figure preparation, writing the final manuscript, guarantor. AA, OA-M, AH: data collection, validation, critically reviewed and finally approved the manuscript. TL: data validation, analysis, interpretation of data, drafting tables and figure preparation, critically reviewed and approved the final manuscript. MA-H: supervision, project administration and planning, data validation, critically reviewed and approved the final draft.

Funding This work was supported by Princess Nourah bint Abdulrahman University Researchers Supporting Project number (PNURSP2022R118), Princess Nourah bint Abdulrahman University, Riyadh, Saudi Arabia.

Competing interests None declared.

\section{Patient consent for publication Not required.}

Ethics approval The study was approved by the institutional review board at Princess Nourah bint Abdulrahman University (IRB log number 20-0383).

Provenance and peer review Not commissioned; externally peer reviewed.

Data availability statement Data are available upon reasonable request, and approval of the IRB at Princess Nourah bint Abdulrahman University.

Open access This is an open access article distributed in accordance with the Creative Commons Attribution Non Commercial (CC BY-NC 4.0) license, which permits others to distribute, remix, adapt, build upon this work noncommercially, and license their derivative works on different terms, provided the original work is properly cited, an indication of whether changes were made, and the use is non-commercial. See: http://creativecommons.org/ licenses/by-nc/4.0/.

\section{ORCID iD}

Maha Al-Mohaissen http://orcid.org/0000-0002-5145-0648

\section{REFERENCES}

1 Bui QM, Daniels LB. A review of the role of breast arterial calcification for cardiovascular risk stratification in women. Circulation 2019;139:1094-101.

2 Parikh RV, Iribarren C, Lee C, et al. Kidney function, proteinuria and breast arterial calcification in women without clinical cardiovascular disease: the Minerva study. PLoS One 2019;14:e0210973.

3 Nazari SS, Mukherjee P. An overview of mammographic density and its association with breast cancer. Breast Cancer 2018;25:259-67.

4 Advani SM, Zhu W, Demb J, et al. Association of breast density with breast cancer risk among women aged 65 years or older by age group and body mass index. JAMA Netw Open 2021:4:e2122810-e10.

5 Whelton PK, Carey RM, Aronow WS, et al. 2017 ACC/AHA/AAPA/ABC/ACPM/ AGS/APhA/ASH/ASPC/NMA/PCNA guideline for the prevention, detection, evaluation, and management of high blood pressure in adults: a report of the American College of Cardiology/American heart association Task force on clinical practice guidelines. Hypertension 2018;71:e13-115.

6 Arnett DK, Blumenthal RS, Albert MA, et al. 2019 ACC/AHA guideline on the primary prevention of cardiovascular disease: Executive summary: a report of the American College of Cardiology/American heart association Task force on clinical practice guidelines. Circulation 2019;140:e563-95.

7 American Diabetes Association. 2. Classification and Diagnosis of Diabetes: Standards of Medical Care in Diabetes-2021. Diabetes Care 2021;44:S15-33.

8 American College of Radiology. ACR BI-RADS atlas 5th edition. II. reporting system, 2013. Available: https://www.acr.org/-/media/ACR/Files/RADS/BIRADS/Mammography-Reporting.pdf

9 Demetri-Lewis A, Slanetz PJ, Eisenberg RL. Breast calcifications: the focal group. AJR Am J Roentgenol 2012;198:W325-43.

10 Tehranifar P, Protacio A, Schmitt KM, et al. The metabolic syndrome and mammographic breast density in a racially diverse and predominantly immigrant sample of women. Cancer Causes Control 2015;26:1393-403.

11 lyengar NM, Zhou XK, Mendieta $\mathrm{H}$, et al. Effects of adiposity and exercise on breast tissue and systemic Metabo-Inflammatory factors in women at high risk or diagnosed with breast cancer. Cancer Prev Res 2021;14:541-50.

12 Jabłonowska-Lietz B, Wrzosek M, Włodarczyk M, et al. New indexes of body fat distribution, visceral adiposity index, body adiposity index, waist-to-height ratio, and metabolic disturbances in the obese. Kardiol Pol 2017;75:1185-91.

13 Lin Y-A, Chen Y-J, Tsao Y-C, et al. Relationship between obesity indices and hypertension among middle-aged and elderly populations in Taiwan: a community-based, cross-sectional study. BMJ Open 2019;9:e031660.

14 Ross R, Neeland IJ, Yamashita S, et al. Waist circumference as a vital sign in clinical practice: a consensus statement from the IAS and ICCR Working group on visceral obesity. Nat Rev Endocrinol 2020;16:177-89.

15 Wu X, Li B, Lin W-Q, et al. The association between obesity indices and hypertension: which index is the most notable indicator of hypertension in different age groups stratified by sex? Clin Exp Hypertens 2019;41:373-80. 
16 Powell-Wiley TM, Poirier P, Burke LE, et al. Obesity and cardiovascular disease: a scientific statement from the American heart association. Circulation 2021;143:e984-1010.

17 Kothari C, Diorio C, Durocher F. The importance of breast adipose tissue in breast cancer. Int J Mol Sci 2020;21:5760.

18 Alhallak I, Wolter KG, Castro Munoz A, et al. Breast adipose regulation of premenopausal breast epithelial phenotype involves interleukin 10. J Mol Endocrinol 2021;67:173-88.

19 Rice MS, Biessy C, Lajous M, et al. Metabolic syndrome and mammographic density in Mexican women. Cancer Prev Res 2013;6:701-10.

20 Martínez-Arroyo A, Moreno-Macías H, Scalabrino AP, et al. Metabolic syndrome and mammographic density in premenopausal Chilean women. Nutr Cancer 2017:69:254-60.

21 Pekcan MK, Findik RB, Tokmak A, et al. The relationship between breast density, bone mineral density, and metabolic syndrome among postmenopausal Turkish women. J Clin Densitom 2020;23:490-6.

22 Kim B-K, Chang Y, Ahn J, et al. Metabolic syndrome, insulin resistance, and mammographic density in pre- and postmenopausal women. Breast Cancer Res Treat 2015;153:425-34.

23 Kankoon N, Somboonporn W, Soontrapa S. Mammographic density and metabolic syndrome in climacteric women. Thai Journal of Obstetrics and Gynaecology 2014;22:95-102.

24 Tehranifar P, Reynolds D, Fan X, et al. Multiple metabolic risk factors and mammographic breast density. Ann Epidemiol 2014;24:479-83.

25 Salem C, Atallah D, Safi J, et al. Breast density and breast cancer incidence in the Lebanese population: results from a retrospective multicenter study. Biomed Res Int 2017;2017:1-9.

26 Sprague BL, Gangnon RE, Burt V, et al. Prevalence of mammographically dense breasts in the United States. J Nat/ Cancer Inst 2014;106. doi:10.1093/jnci/ dju255. [Epub ahead of print: 1209 2014].

27 Al-Mousa DS, Alakhras M, Spuur KM, et al. Mammographic breast density profile of Jordanian women with normal and breast cancer findings. Breast Cancer 2020;14:117822342092138.

28 Jo H-M, Lee EH, Ko K, et al. Prevalence of women with dense breasts in Korea: results from a nationwide cross-sectional study. Cancer Res Treat 2019;51:1295-301.

29 Yang Y, Liu J, Gu R, et al. Influence of factors on mammographic density in premenopausal Chinese women. Eur J Cancer Prev 2016;25:306-11.

30 Al-Nozha MM, Arafah MR, Al-Maatouq MA, et al. Hyperlipidemia in Saudi Arabia. Saudi Med J 2008;29:282-7.

31 Alotaibi A, Perry L, Gholizadeh L, et al. Incidence and prevalence rates of diabetes mellitus in Saudi Arabia: an overview. J Epidemiol Glob Health 2017;7:211-8.

32 Ebbert JO, Jensen MD. Fat depots, free fatty acids, and dyslipidemia. Nutrients 2013;5:498-508.

33 Kotsis V, Jordan J, Micic D, et al. Obesity and cardiovascular risk: a call for action from the European Society of hypertension Working group of obesity, diabetes and the high-risk patient and European association for the study of obesity: Part A: mechanisms of obesity induced hypertension, diabetes and dyslipidemia and practice guidelines for treatment. J Hypertens 2018;36:1427-40.

34 Hosseini A, Khoury AL, Varghese F, et al. Changes in mammographic density following bariatric surgery. Surg Obes Relat Dis 2019;15:964-8.

35 Paccaud F, Schlüter-Fasmeyer V, Wietlisbach V, et al. Dyslipidemia and abdominal obesity: an assessment in three general populations. J Clin Epidemiol 2000;53:393-400.

36 Momin M, Fan F, Li J, et al. Joint effects of body mass index and waist circumference on the incidence of hypertension in a community-based Chinese population. Obes Facts 2020;13:245-55.

37 Zhang W, He K, Zhao H, et al. Association of body mass index and waist circumference with high blood pressure in older adults. BMC Geriatr 2021;21:260.

38 Goossens GH. The metabolic phenotype in obesity: fat mass, body fat distribution, and adipose tissue function. Obes Facts 2017;10:207-15.

39 Abu-Shahba N, Mahmoud M, Abdel-Rasheed M, et al. Immunomodulatory and antioxidative potentials of adipose-derived mesenchymal stem cells isolated from breast versus abdominal tissue: a comparative study. Cell Regen 2020;9:18.

40 Faulkner JL, Belin de Chantemèle EJ. Sex differences in mechanisms of hypertension associated with obesity. Hypertension 2018;71:15-21.

41 Zore T, Palafox M, Reue K. Sex differences in obesity, lipid metabolism, and inflammation-A role for the sex chromosomes? Mol Metab 2018;15:35-44.

42 Warren TY, Wilcox S, Dowda M, et al. Independent association of waist circumference with hypertension and diabetes in African American women, South Carolina, 2007-2009. Prev Chronic Dis 2012;9:E105-E05.

43 Buschard K, Thomassen K, Lynge E, et al. Diabetes, diabetes treatment, and mammographic density in Danish diet, cancer, and health cohort. Cancer Causes Control 2017;28:13-21.

44 Miller B, Chalfant H, Thomas A, et al. Diabetes, obesity, and inflammation: impact on clinical and radiographic features of breast cancer. Int J Mo/ Sci 2021;22. doi:10.3390/ijms22052757. [Epub ahead of print: 09 Mar 2021].

45 Suvannarerg V, Claimon T, Sitthinamsuwan P, et al. Clinical, mammographic, and ultrasonographic characteristics of diabetic mastopathy: a case series. Clin Imaging 2019;53:204-9.

46 Lanzer P, Hannan FM, Lanzer JD, et al. Medial arterial calcification: JACC stateof-the-art review. J Am Coll Cardiol 2021;78:1145-65.

47 Avogaro A, Fadini GP. Mechanisms of ectopic calcification: implications for diabetic vasculopathy. Cardiovasc Diagn Ther 2015;5:343-52.

48 Lee SC, Phillips M, Bellinge J, et al. Is breast arterial calcification associated with coronary artery disease?-A systematic review and meta-analysis. PLoS One 2020;15:e0236598.

49 Hendriks EJE, de Jong PA, van der Graaf Y, et al. Breast arterial calcifications: a systematic review and meta-analysis of their determinants and their association with cardiovascular events. Atherosclerosis 2015;239:11-20.

50 Odink AE, van der Lugt A, Hofman A, et al. Risk factors for coronary, aortic arch and carotid calcification; the Rotterdam study. J Hum Hypertens 2010;24:86-92. 